\title{
Determination of instability of a DP 980 steel sheet under different stress states based on experiment and theoretical models
}

\author{
Hong-Wu Song ${ }^{1,}$, Dong-Zhi Sun ${ }^{2}$, Florence Andrieux ${ }^{2}$ and Shi-Hong Zhang ${ }^{1}$ \\ ${ }^{1}$ Institute of Metal Research, Chinese Academy of Sciences, 110016 Shenyang, China \\ ${ }^{2}$ Fraunhofer Institute for Mechanics of Materials IWM, 79108 Freiburg, Germany
}

\begin{abstract}
It is generally accepted that the formability of sheet metal is limited by the onset of instability which can be described by forming limit diagram (FLD) in $\varepsilon_{1}-\varepsilon_{2}$ space. Common tests for FLD data usually cover strain ratio range from uniaxial to equal biaxial stretching, $-1 / 2 \leq \varepsilon_{2} / \varepsilon_{1} \leq 1$. In this paper a new design of cruciform type specimen for uniaxial stretching is proposed to test formability with strain ratios in the range from shear to tension, $-1 \leq \varepsilon_{2} / \varepsilon_{1} \leq$ $1 / 2$ which can be controlled by the geometry parameters. The forming limit of a DP 980 steel sheet was determined using both conventional method and proposed specimen with strain ratios from $-1 \leq \varepsilon_{2} / \varepsilon_{1} \leq 1$. Then the experimental results were drawn in both $\varepsilon_{1}-\varepsilon_{2}$ and $\varepsilon_{\mathrm{eq}}-\sigma_{\mathrm{m}} / \sigma_{\mathrm{eq}}$ spaces, and the predicted results based on Hill, Swift, M-K, and Hora instability models were compared with the experimental ones. The formability features of the studied steel in whole strain ratio range and differences among the investigated theoretical models were finally discussed. The results indicate that the M-K instability model shows better prediction of the studied steel compared to the other models investigated in this research.
\end{abstract}

\section{Introduction of theoretical models and experimental methods for sheet metal formability}

It is generally accepted that the formability of sheet metal is limited by the onset of instability which can be described by forming limit diagram (FLD) which concept was published by Keeler [1] and then developed by Goodwin [2] almost 50 years ago. During the last decades different assessment methods for determination of metal sheet formability have been developed.

Many theoretical models are proposed and are presented in detail (formulation of the model, solving methods, numerical aspects, advantages and limitations) in [3]. Some of the well-established theoretical models which will be used in this paper are briefly introduced in the following.

The first models proposed by Swift [4] and Hill [5] assuming homogeneous sheet metals were so-called models of diffuse necking and localized necking, respectively. Hill suggested the onset of localized necking when the increase of stress by strain hardening is balanced by geometrical softening due to thickness reduction. Swift assumed the onset of diffuse necking occurs when the load reaches maximum in both principal directions. According to Hill approach the localized neck is expected along a line of zero extension and a strain increment has to be negative. The Swift criterion is considered to underestimate the formability for negative minor strain. So both criteria are commonly combined together with the Hill criterion for the left side of the FLD and Swift model for the right one.

Later on, the Swift model has been further developed by Hora and Tong named Modified Maximum Force Criterion (MMFC) [6] for theoretical prediction of the localized necking considering the additional hardening effect to postpone localization by the stress transformation, and it was referred as Hora model in this paper.

Marciniak and Kuczynski [7] proposed a model taking into account the geometrical nonhomogenity of the sheet metal, known as M-K method. The M-K model proposed a physical approach by introducing the idea of flow localization in presence of an initial imperfection.

The mentioned theoretical models have their own advantages and limitations which are described in detail in [3]. However, there is still lack of systemically study on their applicability for different kind of materials. In this investigation, comparative research will be carried out to investigate the difference for forming limit prediction given by different theoretical models for a DP 980 steel.

Over the years, many researchers also studied on experimental determination methods for FLD. Some of these tests are summarised in Fig. 1 [8]. The most established testing procedure to determine FLD was proposed by Nakajima et al. [9]. A hemi-spherical punch and several specimen geometries are used for testing material formability under different strain paths.

\footnotetext{
$\bar{a}$ Corresponding author: hwsong@imr.ac.cn
} 


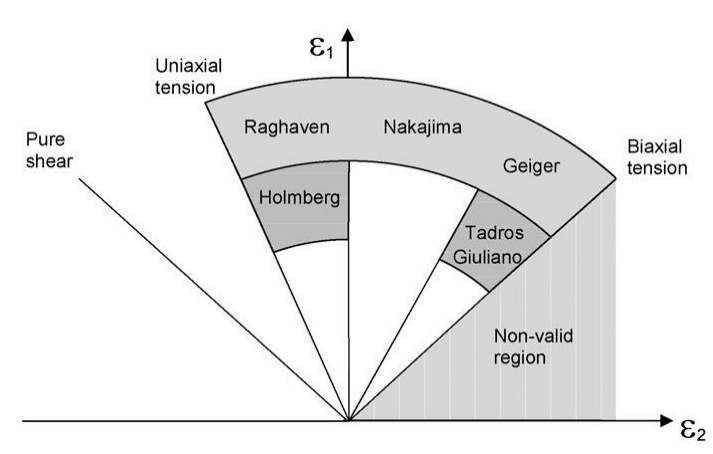

Figure 1. Summary of standard sheet material testing procedures with tested path range

However, as is shown in Figure 1, the standard methods for testing FLD are mainly used to characterise formability under certain in-plane load conditions from uniaxial tension, through plane-strain to biaxial stretching. But in industrial forming processes not only in-plane loads, but a mixture of in-plane, pure-shear and combined shear-and tension loads can also be observed [8, 10]. Today there is still a lack in testing equipment for those combined load conditions because with conventional testing set-ups no superposed shear rates can be realized.

Even for uniaxial tension it is not easy to achieve a constant loading path from onset of instability to rupture due to the necking effects observed in most steels. Smooth tensile specimen with a hole in the specimen center is very often used to approximate the stress state of uniaxial tension [11]. However, the disadvantage of the specimen is that the strain field in the specimen is very inhomogeneous and only a small region near the hole shows the stress state close to the uniaxial tension.

Although biaxial loading with cruciform specimens can be used to obtain shear-tension strain paths [12], the set-up is so complex and not easy to handle.

Recently, Shouler et al. [8] and Held et al. [10] proposed similar type of samples for characterising material formability under shear-tension loads with relatively simple set-up. However, the obtained strain paths are almost pure shear, which not easily occur during forming process. In addition, for advanced high strength steels such as DP steels fracture happens normally without instability under pure shear loading.

Considering the possible strain paths during forming process with mainly tension superimposed with some shear components, new sample geometry based on cruciform specimen is proposed to test formability under shear-tension strain paths which are closer to uniaxial tension. The set-up is only uniaxial tension loading, so the main advantage of the proposed testing is easy to handle and the measurements can also be easy to repeat.

\section{FLD obtained by standard Nakajima testing and comparison with different theoretical models}

For a DP 980 steel sheet with a thickness $1.5 \mathrm{~mm}$, the standard Nakajima testing-DIN EN ISO 12004-2 was applied to measure the FLD and the failure curve [13, 14].
Instability limits under different strain paths were achieved by changing the notched width of the specimen as shown in Figure 2. Different theoretical models including Swift, Hill, Hora and M-K models which were briefly described in the introduction part were applied to predict the formability. A Von Mises isotropic yield function and a Swift power law hardening equation were used. For M-K model, the initial imperfection parameter $f_{0}$ is fixed to 0.995 calculated by surface roughness of the delivered sheets using methods proposed in [15].

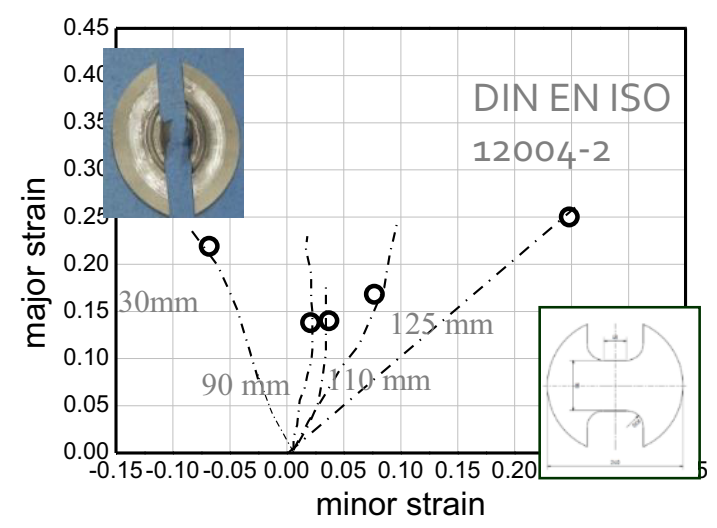

Figure 2. Measured FLD data by standard Nakajima testing [13]
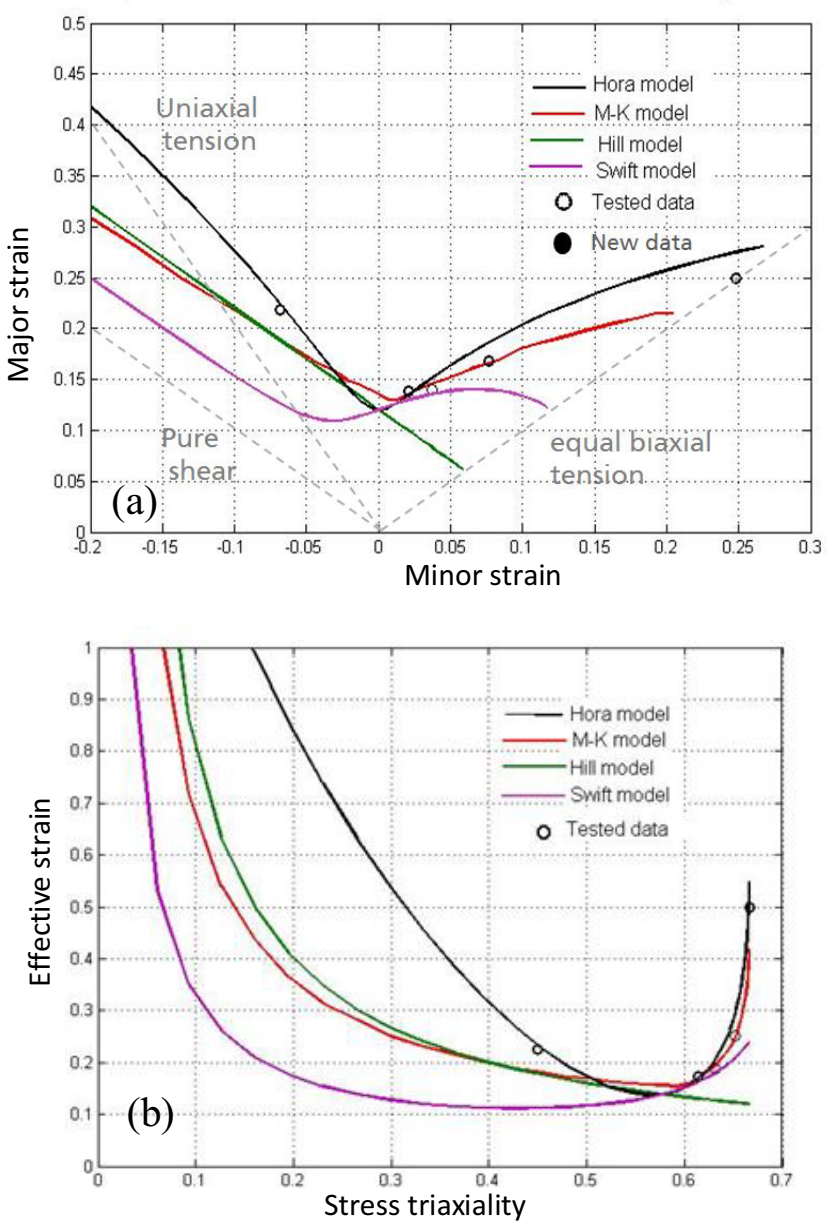

Figure 3. Comparison of FLD data by standard Nakajima testing and different theoretical models a) in the major strainminor strain space, b) in the effective strain-stress triaxiality space 
Comparison of tested data and prediction results in both major strain-minor strain space and effective strainstress triaxiality space is given in Figure 3. The triaxiality is defined as the ratio of the mean stress $\sigma_{m}$ to the von Mises stress $\sigma_{e q}$.

It can be seen that in the right side of FLD, the Hora and $\mathrm{M}-\mathrm{K}$ models predict similar results and have good agreement with tested data. The Swift model obviously underestimates the formability and the Hill model is not relevant on the right side. In the left side, remarkable difference occurs among the theoretical models. However, as there is only one tested data in the left side of FLD, it is difficult to say which model is more applicable for formability prediction of the studied steel. As suggested in [15] the effective strain related FLD (shown in Figure 3 (b)) is less strain path dependent than the traditional FLD in major strain-minor strain space (shown in Figure 3 (a)), the effective related FLD is more suitable for practical forming process. As there is obvious difference of the theoretical FLD models on the left side, more testing data are needed to make better comparison and evaluation of the different theoretical models.

\section{Proposed instability tests for shear- tension strain paths}

\subsection{Design of a new specimen for instability test under shear-tension strain path}

Normal uniaxial tension loading can be used to get sheartension strain path with the proposed specimen with a geometry given in Figure 4. It is a modified cruciform specimen which only needs uniaxial loading. The strain paths in the specimen can be changed by adjusting the three dimensional parameters $W, R, t$ shown in Figure 4.

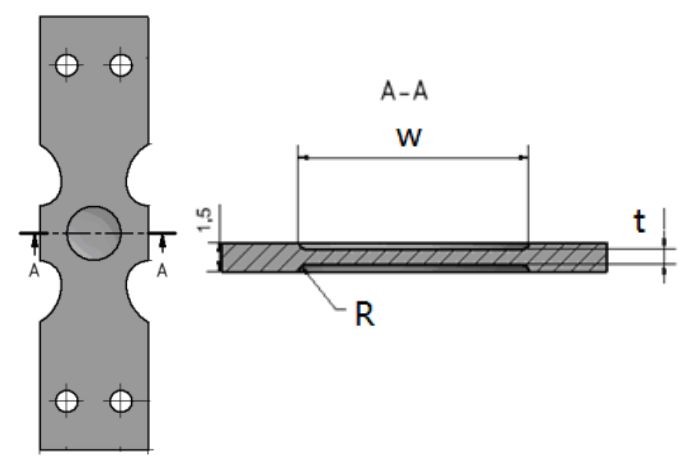

Figure 4. The geometrical design of the proposed specimen

The test on the new specimen is modelled with LSDyna using solid elements with edge length of $0.5 \mathrm{~mm}$. The same yield function and hardening law as for theoretical prediction of FLD are used. The distribution and evolution of thickness strain were examined during deformation and the most thinned region in the specimen can be considered where to be the location of the onset of instability. The simulated results of strain paths in the most thinning region for different specimens are shown in Figure 5. These two regions are located in the center and at the notch. It can be seen that, the strain paths in the region where instability can possibly occur lie under shear-tension (near the specimen center) or near uniaxial tension (near the notch) dependent on where instability occurs. The position for the onset of instability can be controlled by adjusting the dimension parameters. All the predicted strain paths are linear in the major strain-minor strain space and almost linear in the effective strain-stress triaxiality space. These verified the applicability of the propose specimen test.

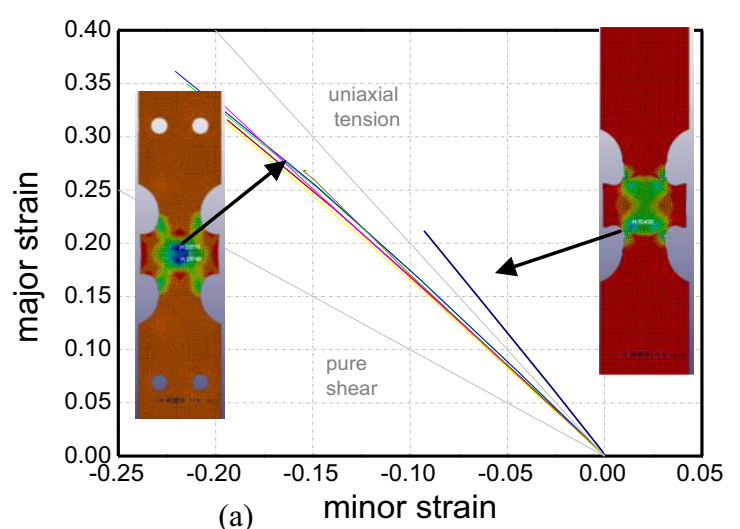

(a)

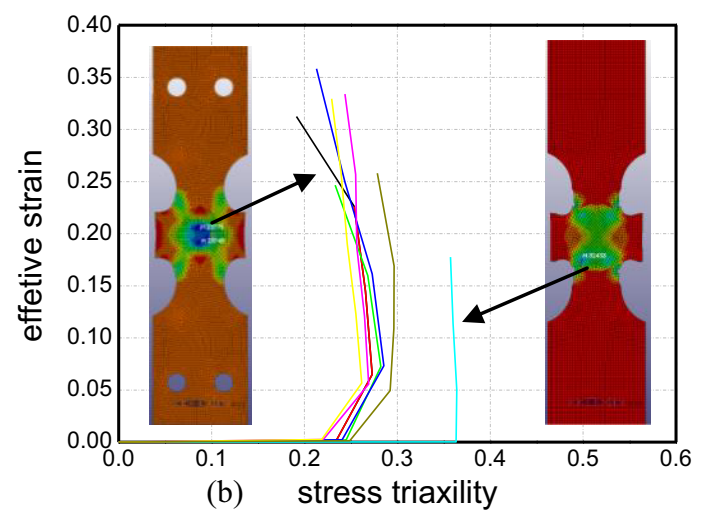

Figure 5. Simulated results for strain paths in the most thinned region in the specimen during uniaxial tension loading a) in the major strain-minor strain space, b) in the effective strain-stress triaxiality space

\subsection{Experimental evaluation of the proposed tests}

Several specimens with different geometries were extracted are prepared with different dimension parameters by sparkling cutting. Static uniaxial tension tests are carried out and the DIC (Digital image correlation) technique based on using ARAMIS system is applied to measure strain evolution during deformation.

Figure 6 shows the measured load-displacement curves for different specimen geometries and the typical broken specimens, and the dashed line is the simulation results of specimen 1 which indicates good agreement with experiment data

The thickness reductions were evaluated and the instability occurrence is defined as follows:

First, according to the measured distribution of thickness reduction as shown in Figure 7 (a) and Figure 7 (c), the most thinning regions with higher thinning can be captured. 
Then points are selected across the thinned regions to evaluate the evolution of thickness reductions as shown correspondingly in Figure 7 (b) and Figure 7 (d). Finally when thickness reduction of at one point starts to increase faster than at the nearly ones from both side, the instability onset is assumed. .

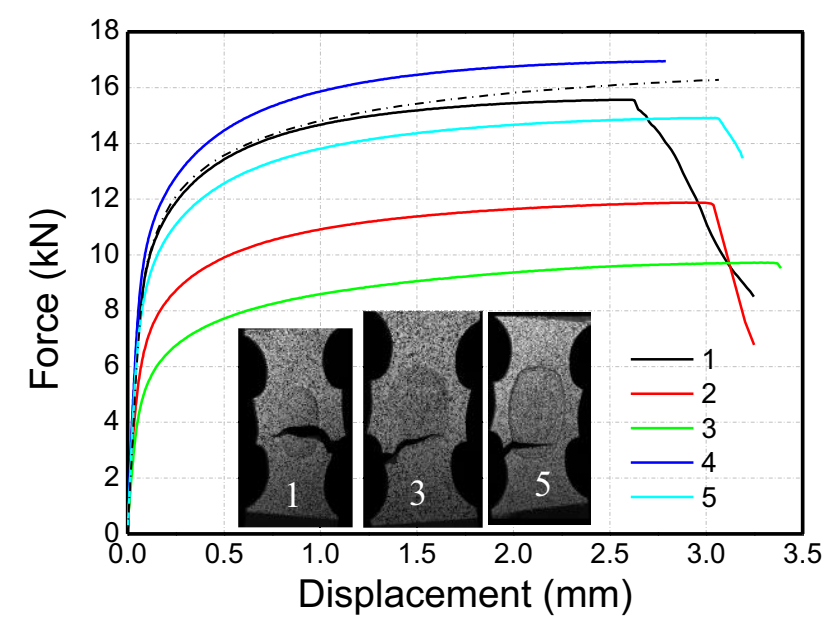

Figure 6. Measured load-displacement curves for different specimen geometries and the typical broken specimens.

The thickness reductions were evaluated and the instability occurrence is defined as follows:

First, according to the measured distribution of thickness reduction as shown in Figure 7 (a) and Figure 7 (c), the most thinning regions with higher thinning can be captured.

Then points are selected across the thinned regions to evaluate the evolution of thickness reductions as shown correspondingly in Figure 7 (b) and Figure 7 (d).

And finally when thickness reduction of at one point starts to increase faster than at the nearly ones from both side, the instability onset is assumed.

The defined instability occurrence for two specimens are given as examples indicated by dot line in Figure 7 (b) and Figure 7 (d) for different positions where instability occurs at points 2,3 and point 0 respectively.

Using this definition, five specimens with different dimension parameters are tested, and the measured strain paths and strains for instability in both traditional major stain-minor strain space and effective strain-stress triaxiality space are given in Figure 8. It can be clearly seen that as FE predicted, all the tested strain paths where instability occurs are almost linear and lie in sheartension or near uniaxial tension dependent on the position where instability occur controlled by specimen dimensional parameters. It can also be found that sheartension strain paths were achieved when fracture failure initiates near the centre of the specimens and near uniaxial tension strain paths were achieved when fracture failure initiates at the edge notches.

The failure can be controlled by adjusting dimensional parameters of tested specimens.
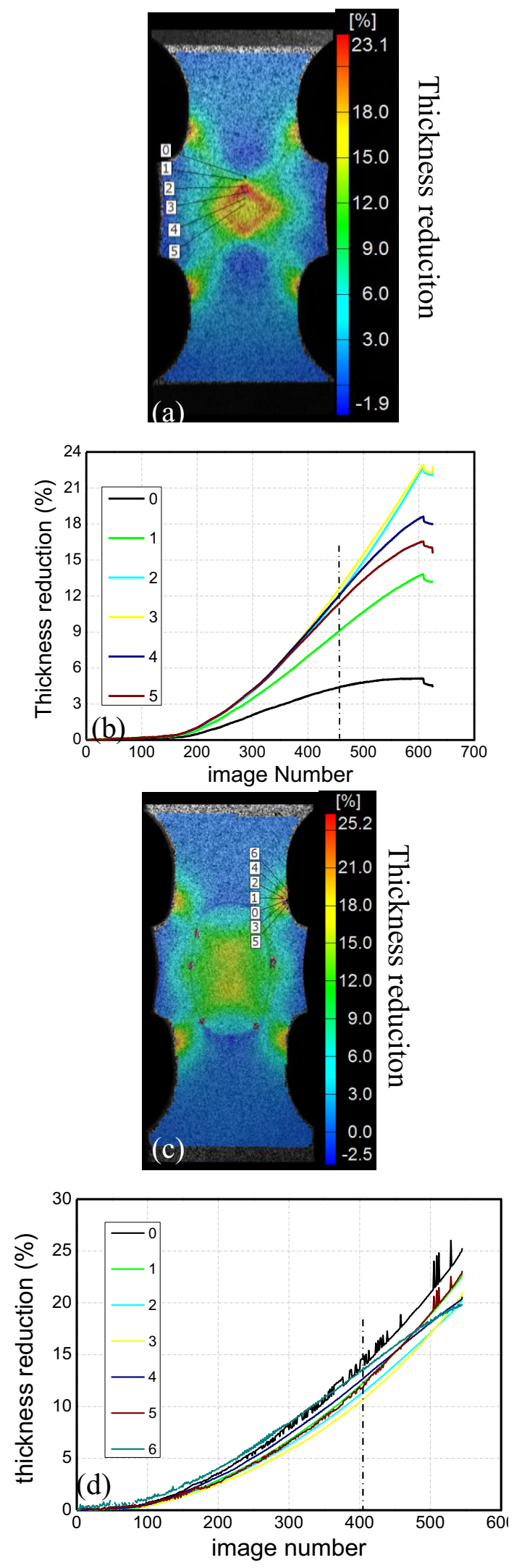

Figure 7. Definition of instability occurrence in the tested specimens a) distribution of thickness reduction of specimen 1 , b) evolution of thickness reduction of selected point in specimen 1, c) distribution of thickness reduction of specimen 2 and d) evolution of thickness reduction of selected point in specimen 2 

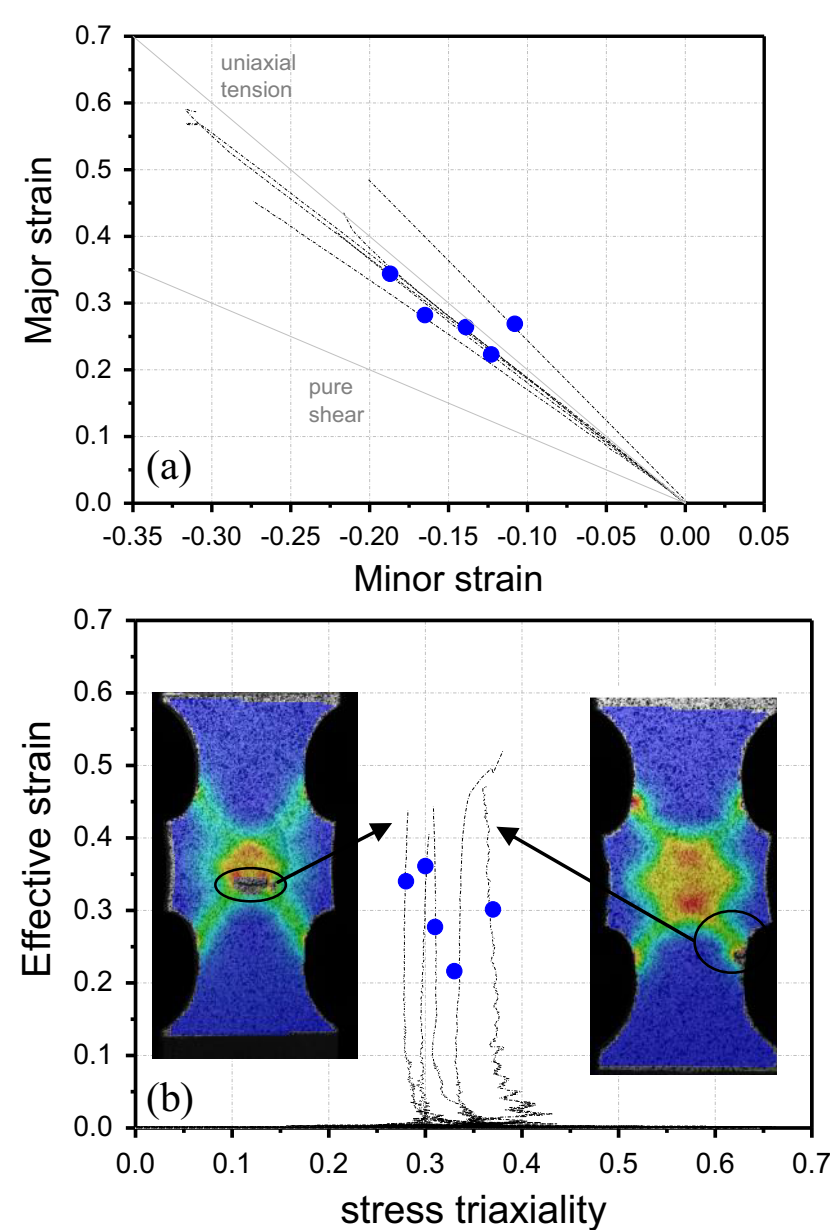

Figure 8. Measured instability results in both a) traditional FLD and b) effective related space

\section{Discussions}

Each of the five specimens used to get results in Figure. 8 are tested twice for repeatable evaluation. And the measured instability results from the ten tests (named new data) and from the standard Nakajima tests (named tested data) were drawn together in both traditional FLD and effective strain-stress triaxiality space, which are drawn in Figure 9. The comparison of the predictions by the investigated theoretical models are also presented in the figure.

With the help of new testing data, an evaluation of the theoretical models in the left side of the FLD can be done. The M-K and Hill models seem to give better formability prediction for the studied DP 980 steel in region between shear and uniaxial tension. Hora and Swift model respectively overestimates or underestimates the formability.

In order to confirm this result other variations of the proposed specimen geometry have to be determined in order to cover the whole range between shear and uniaxial tension with experimental results.
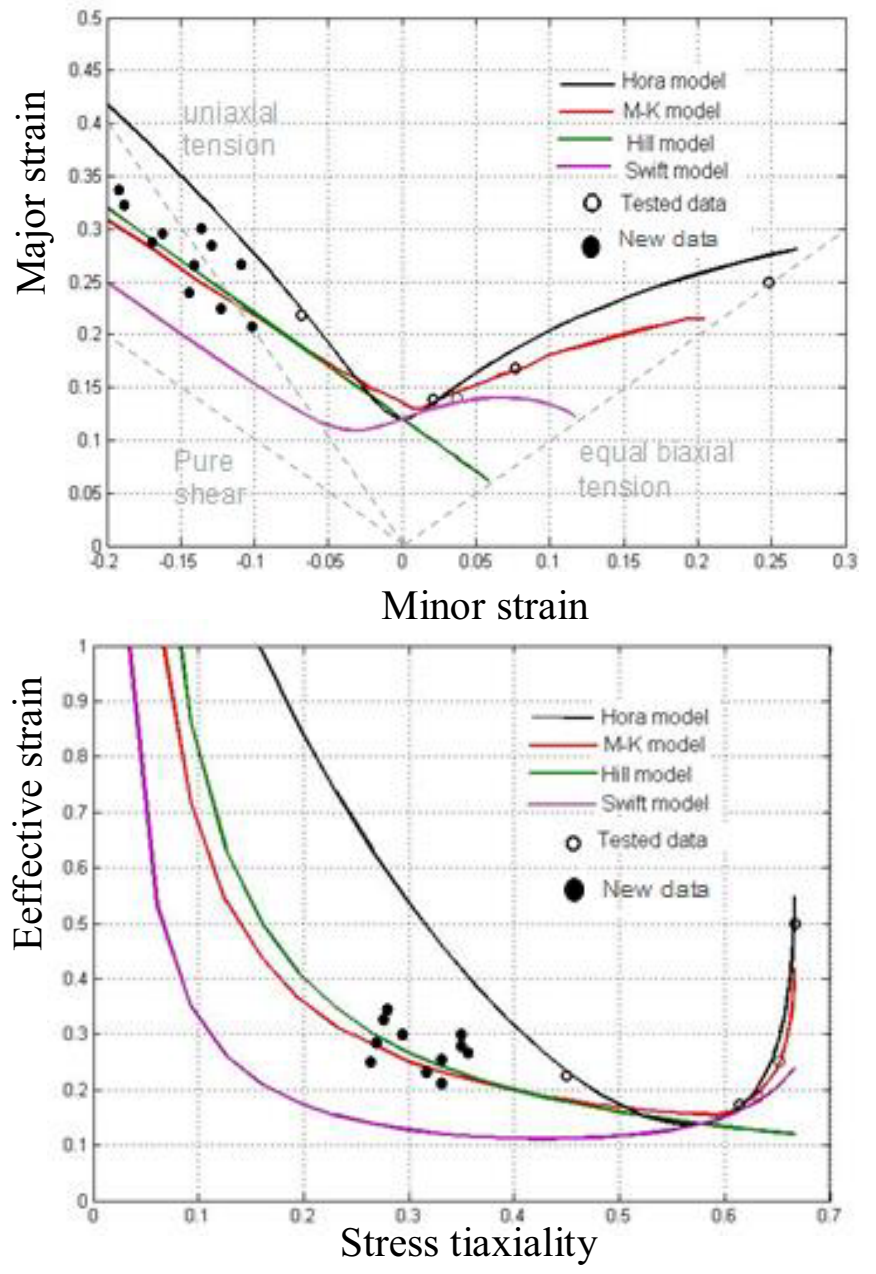

Figure 9. The comparison of measured instability results by proposed testing with different theoretical models in both traditional FLD and effective related space

\section{Summaries}

A new testing method was proposed for formability measurement under shear-tension strain path using a simple experimental set up (uniaxial tension). Both FE simulation and experimental evaluations validated the applicability of the proposed testing. The comparison of tested formability with different theoretical prediction for the studied DP 980 steel shows that the M-K model gives better results especially under shear-tension region in the less path dependent effective strain-stress triaxiality space.

\section{Acknowledgement}

The support of CSC (Chinese scholarship council) is greatly appreciated.

\section{References}

1. S. P. Keeler, W. A. Backofen, Trans. ASM 56, 25 (1963)

2. G. M. Goodwin, Society of Automotive Engineers No. 680093, 380 (1968) 
3. D. Banabic, Sheet Metal Forming Processes, (Springer, Heidelberg, 2010)

4. H. W. Swift, J. Mech. Phys. Sol. 1, 1 (1952)

5. R. Hill, J. Mech. Phys. Sol. 1, 19 (1952)

6. P. Hora, L. Tong, Proc. of the IDDRG Congress. (Lisbon, 1994)

7. Z. Marciniak, K. Kuckzynski, Int. J. Mech. Sci. 9, 609 (1967)

8. D. R. Shouler, J. M. Allwood, J. Mater. Proc. Tech. 210, 1304 (2010)

9. K. Nakajima, T. Kikuma, K. Hasuka, Proc. of the 7th Biennial IDDRG Congress. (Amsterdam, 1972)

10. C. Held, R. Schleich, M. Sindel, M. Liewald, Int. J. Mater. Form. 2, 467 (2009)

11. A. Trondl, S. Klitschke, W. Böhme, D.-Z. Sun, Fraunhofer IWM 2015 Schlussbericht, IWMBerichts-Nr.: 1235/2015, Stiftungs-Nr. S24/10195/12

12. A. Hannon, P. Tiernan, J. Mater. Proce. Tech. 198, 1 (2008)

13. S. Klitschke, W. Böhme, J. Schüler, J. Skoeries, D. Neumann, AVIF-Projekt (Stiftungs-Nr. S24/10195/12), Zwischenbericht PbA-Sitzung am 18.03.2015

14. S. Klitschke, W. Böhme, Tagungsband Werkstoffprüfung S. 213-218, (2014)

15. M. Nurcheshmeh, D. E. Green, Int. J. Mater. Form. 7, 1 (2014) 